\title{
Organised macular plaques in exudative diabetic maculopathy
}

\author{
RAGNAR SIGURDSSON AND IAIN S. BEGG \\ From the Department of Ophthalmology, University of British Columbia, and \\ Vancouver General Hospital, Vancouver, Canada
}

SUMMARY Plaques of hard exudate measuring 0.5 to 1.0 disc diameters, which occurred in 5 eyes of 4 patients with diabetic maculopathy, showed complete organisation in 8 to 18 months with the formation of scars measuring 0.2 to 0.5 disc diameters. The visual acuity was related to the size and situation of the scar within the fovea, and, when the foveola was involved by plaque formation, visual reduction did not progress with subsequent formation of scar tissue. Fluorescein angiograms showed absence of ingrowing choroidal neovascularisation and a discrete residual hyperfluorescence beneath the resolving plaques and within the scar tissue, which resembled a serous detachment of pigment epithelium but may have resulted from a combination of pigment atrophy and leakage from retinal capillaries. This late clinical appearance may be the only ophthalmoscopic evidence that a severe exudative maculopathy has been primarily responsible for severe visual loss, and should be differentiated from cicatricial disciform lesions in older patients. The absence of ingrowing choroidal neovascularisation, subretinal haemorrhage, and pronounced serous retinal detachment substantiates our previous conclusions based on a morphological study from a limited number of sections that the origin of the fibrous replacement was not choroidal but was derived partly from the pigment epithelium and partly from the retinal vasculature. Juxtaposition of a deeply situated hard exudate plaque and the retinal pigment epithelium may initiate focal metaplasia and subsequent organisation with scar formation.

Spontaneous progression and regression of hard exudate in diabetic maculopathy was described by King et al. ${ }^{1}$ and Dobree, ${ }^{2}$ who found that plaque formation required years for resolution and was not followed by visual recovery. In a previous report ${ }^{3}$ we described the pathology of an organised macular plaque in exudative diabetic maculopathy and observed that the fibrous replacement was partly retinovascular and partly pigment epithelial in origin, suggesting that it was a specific pathological entity. The present study reports the resolution of macular plaques accompanied by organisation in 5 eyes of 4 patients with diabetic maculopathy. This late clinical appearance, which is fully described for the first time, may be the only ophthalmoscopic evidence that a severe exudative maculopathy has been primarily responsible for severe visual loss, and should be differentiated from disciform macular degeneration in older patients.

Correspondence to Dr I. S. Begg, Department of Ophthalmology, University of British Columbia, 2550 Willow Street, Vancouver, Canada V5Z 3N9.

\section{Material and methods}

The cases were obtained by reviewing the biomicroscopic descriptions and stereo colour photographs of patients showing exudative diabetic maculopathy with plaques measuring $\frac{1}{4}$ of a disc diameter or greater, who had attended the Diabetic Retinopathy Service during the past 6 years. Only those patients showing the complete evolution from plaque to scar formation have been included in this study. The histological subdivision of macular areas ${ }^{4}$ has been used in the clinical descriptions.

\section{CASE REPORTS}

Case 1. A white woman age 57 was diagnosed as diabetic in June 1974 when a mild background retinopathy was discovered. The visual acuity was $6 / 6$ in both eyes. Diabetes was subsequently well controlled on oral medication. There was no hypertension. In February 1975 she complained of fluctuating vision and micropsia in the right eye which had been present for 1 month. The corrected vision was $6 / 7 \cdot 5$, and there was a plaque of hard 


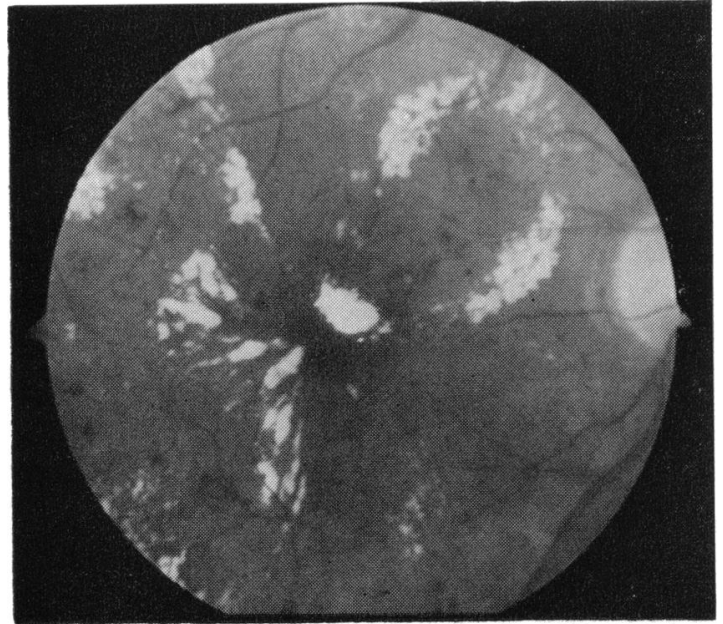

Fig. 1 Case 1, February 1975. Circinate hard exudates and plaque bordering the foveola.

exudate measuring 0.75 disc diameter (DD) bordering the foveola (Fig. 1). Fluorescein angiography showed clusters of microaneurysms, absence of large areas of capillary obliteration, and prominent cystoid retinal oedema within the circinate hard exudate formations. Laser photocoagulation was applied to leaking capillaries in April, May, and August 1975. In July a pigment disturbance was visible within the resolving plaque, and a late angiogram showed a residual hyperfluorescence within the plaque and slight diffuse leakage in the surrounding retina. By October the plaque had

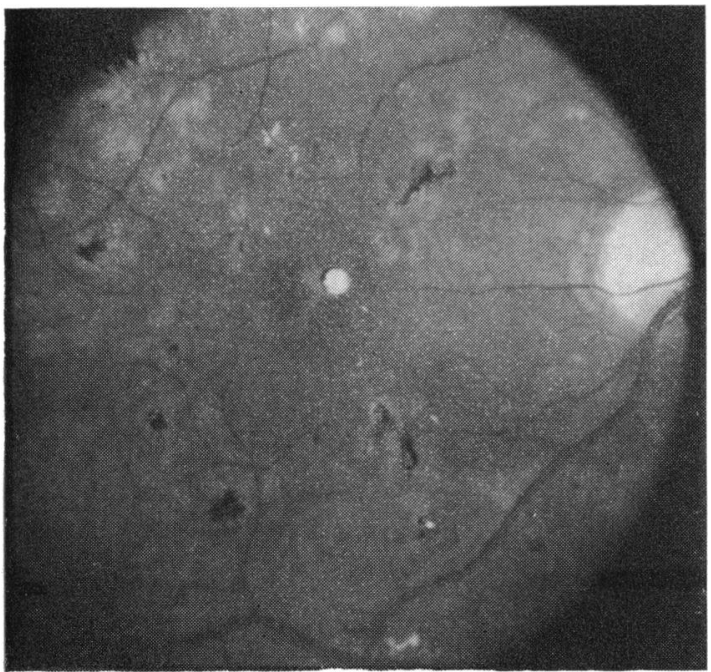

Fig. 2 Case 1, February 1976. Final appearance of the scar. completely resolved, revealing an elevated grey scar at the margin of the foveola, measuring 0.15 DD, and in February 1976 a strand of glial tissue appeared on the surface (Fig. 2). At the last examination, in September 1978, the visual acuity was 6/6, the scar was unaltered, and there was almost complete resolution of hard exudate throughout the macula. Angiography showed an early hyperfluorescence, slight retinal capillary leakage, and late intense discrete leakage and staining within the scar (Fig. 3). A static visual field examination showed markedly reduced retinal sensitivity in this location.

Case 2. A 34-year-old insulin-dependent male diabetic of 12 years' duration was first examined in June 1969 when he complained of bilateral visual

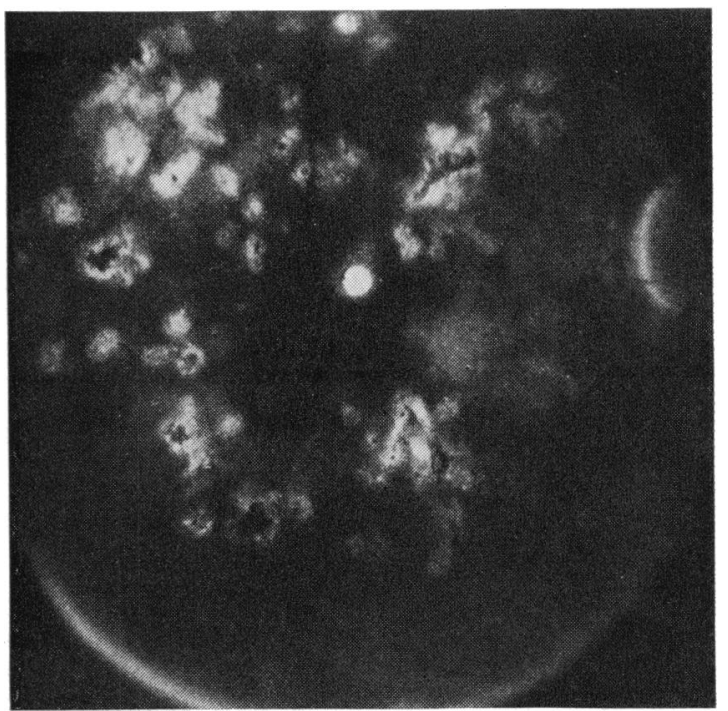

Fig. 3 Case 1, September 1978. Late angiogram shows intense discrete hyperfluorescence and staining within the scar as compared with the control photograph which showed no pseudofluorescence.

deterioration. The blood pressure was normal. The corrected vision was reduced to $6 / 21$ in the right eye and 6/15 in the left eye as a result of a severe exudative diabetic maculopathy. A plaque of hard exudate, $0.75 \mathrm{DD}$, covered the right foveola (Fig. 4). Fluorescein angiography showed a coarse network of dilated tortuous capillaries, numerous diffusely scattered microaneurysms, widespread leakage and cystoid macular oedema. Within a few months vision deteriorated to $6 / 120$ in the right eye and $6 / 60$ in the left eye. He was treated with clofibrate for 1 year. In July 1970 the plaque had cleared in the right eye revealing an underlying elevated pigmentation (Fig. 5). Retinal neovascularisation 
overlying the temporal raphe and elsewhere in the right eye was treated by xenon photocoagulation in February 1971. In July 1973 there was a discrete white scar $0.2 \mathrm{DD}$, with surrounding pigmentation at the right fovea (Fig. 6). Angiography showed an early hyperfluorescence, retinal capillary leakage (Fig. 7), and late comparatively discrete leakage and staining with the scar (Fig. 8). At the last examination, in October 1975, the corrected vision was $6 / 15$ in the right eye and the macular appearance was unchanged. The patient developed an unexplained jaundice and died in October 1975.

Case 3. A 64-year-old white female diabetic patient, controlled on oral medication for 10 years, experienced a gradual visual deterioration in

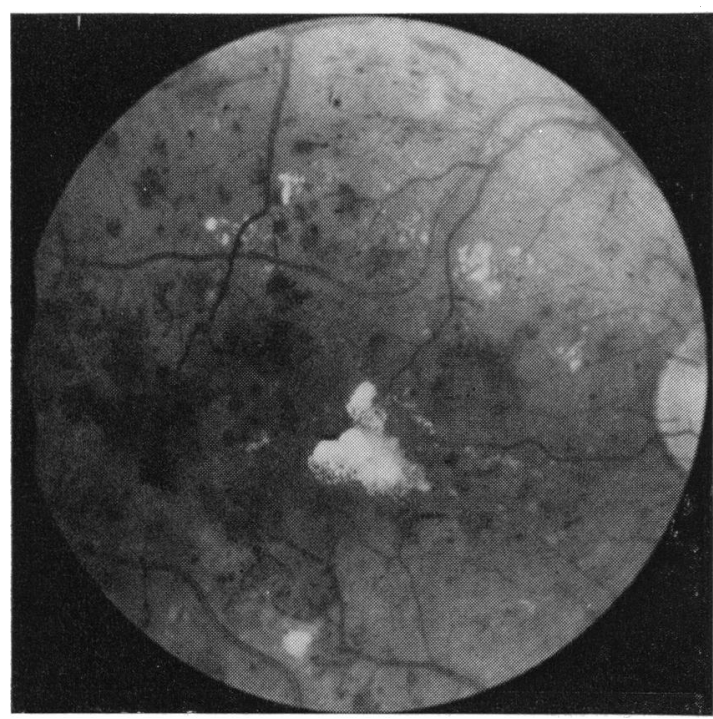

Fig. 4 Case 2, right eye, June 1969. Fundus photograph shows plaque of hard exudate covering the foveola.

her left eye over a 5-month period. She was receiving antihypertensive medication. In March 1974 the corrected vision was $6 / 6$ in the right eye and counting fingers at 1 metre in the left eye. There was a mild background diabetic retinopathy in the right eye, and in the left eye there were circinate formations of hard exudate and a plaque measuring 0.5 DD in the superotemporal fovea, which covered the foveola. Fluorescein angiography showed a marked diffuse capillary leakage, cystoid macular oedema, and late faint hyperfluorescence within the temporal half of the plaque. When she was next examined in September 1974 some circinate hard exudates remained, but the plaque in the left eye had cleared, revealing an elevated pigmented scar, measuring

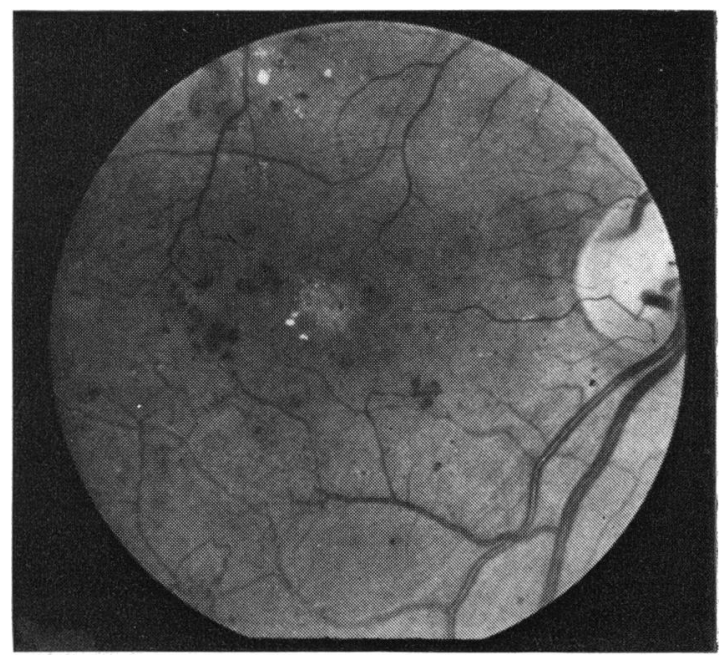

Fig. 5 Case 2, right eye, July 1970. Fundus photograph shows complete resolution of the plaque, revealing underlying elevated pigmentation.

$0 \cdot 25$ DD. The visual acuity was 6/120. Another smaller plaque of hard exudate appeared just nasal to the scar in October 1975. Fluorescein angiography showed an early hyperfluorescence within and surrounding the scar and late discrete hyperfluorescence within the scar. In addition there was marked capillary dilatation with numerous microaneurysms throughout the parafoveal capillary plexus, disruption of the inner border, and pronounced diffuse late leakage from retinal capillaries. The visual

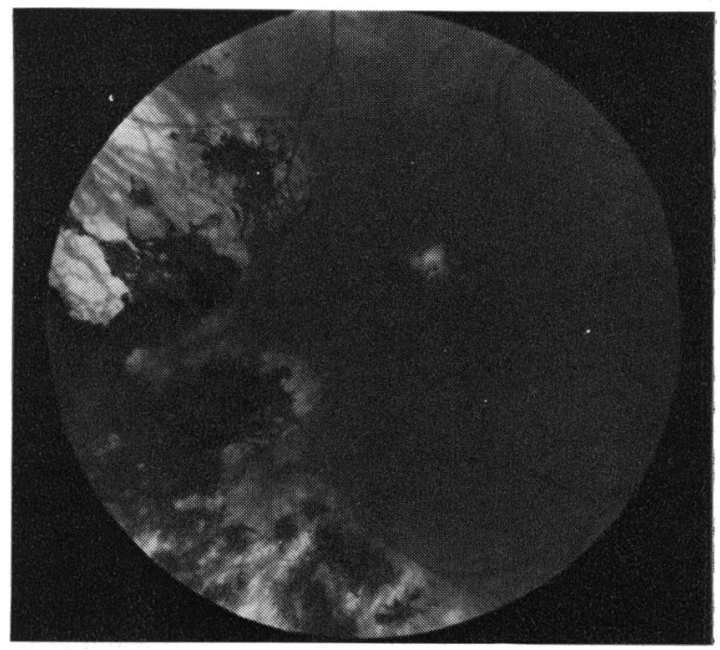

Fig. 6 Case 2, right eye, July 1973. Fundus photograph shows a discrete white scar bordered by pigmentation, and xenon photocoagulation scars temporally. 


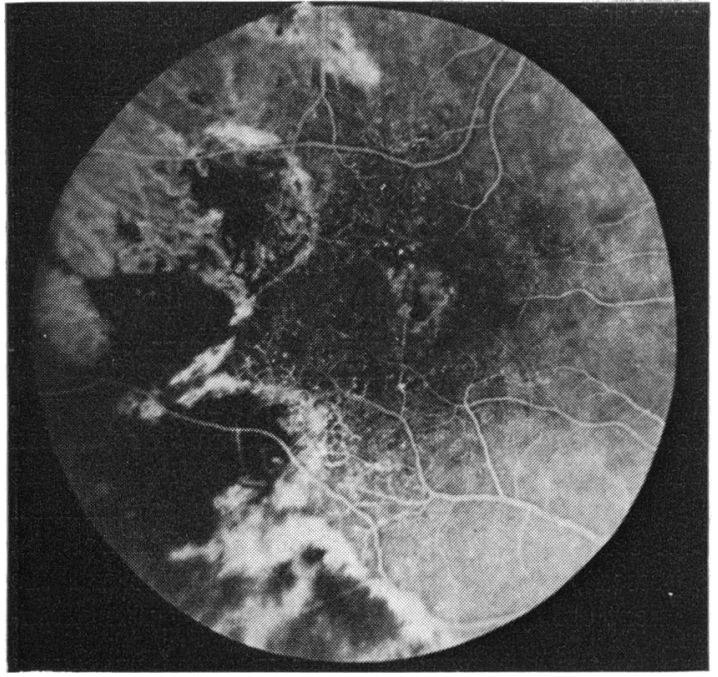

Fig. 7 Case 2, right eye, July 1973. Fluorescein angiogram shows early hyperfluorescence within the scar and diffuse diabetic retinal capillary disease.

acuity and appearance of the scar was unchanged when last examined in June 1976.

Case 4. A 71-year-old white female, who had been diabetic on insulin for at least 13 years, was examined in January 1975 when she complained of a gradual visual deterioration in both eyes of 1 year's duration. The left eye had deteriorated more rapidly in the preceding month. The blood pressure was normal. The corrected vision was $6 / 18$ in the right eye and $6 / 30$ in the left eye, as the result of a severe exudative diabetic retinopathy with many clusters of hard exudate and cystoid macular oedema. Within a month vision deteriorated to $6 / 24$ in the right eye and 6/90 in the left eye, and in view of the recent rapid deterioration laser photocoagulation was applied to leaking capillaries in both maculae. In April the vision was 6/120 in the right eye and $6 / 60$ in the left eye, and there were sheets of hard exudate throughout both maculae. A hard exudate plaque at the fovea, in both eyes, was observed in September 1975. In March 1976 the plaque measured $0.5 \mathrm{DD}$ in the right eye and $1 \mathrm{DD}$ in the left eye. One year later there was considerable resolution of hard exudate in the right eye and the plaque had resolved, revealing a discrete elevated pigmented scar, $0.3 \mathrm{DD}$ in size, with overlying strands of glial tissue. In the left eye large clusters of hard exudate remained within the macula, but the foveal plaque had resolved and was replaced by a discrete elevated pigmented scar measuring 0.75 DD with overlying preretinal gliosis. At the last examination, in March 1978, both eyes showed an elevated grey scar with

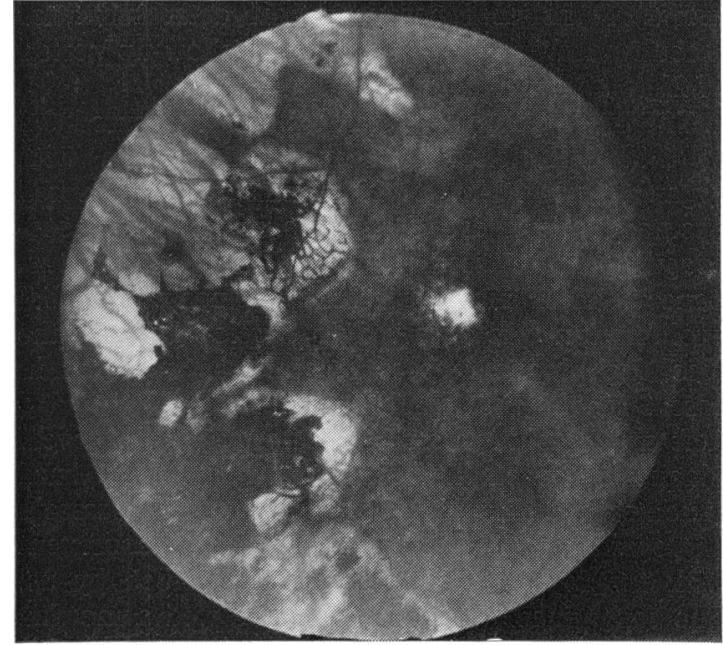

Fig. 8 Case 2, right eye, July 1973. Late angiogram showing residual discrete leakage and staining, as compared to the control photograph, which showed slight pseudofluorescence.

an annulus of pigmentation and slight pigment atrophy, radiating retinal folds, and overlying strands of glial tissue. Angiography showed an early hyperfluorescence and late intense discrete hyperfluorescence within both scars. Throughout both maculae there were multiple areas of capillary obliteration, leaving a coarse network of dilated tortuous capillaries with numerous microaneurysms which showed pronounced late leakage.

\section{Discussion}

Foveal scars developed in 5 eyes of 4 patients who showed typical severe exudative diabetic maculopathy with large waxy hard exudate plaques involving the fovea. The appearance most closely resembled the discrete fibrovascular macular scar described in Coats's disease ${ }^{56}$ and the cicatricial disciform lesion caused primarily by diseases of the choroid. ${ }^{5}$ The scars were round, discrete, slightly elevated, white or grey in colour, and some showed overlying pigment clumps or a thin annulus of pigment surrounded by slight pigment epithelial atrophy. A thin strand of glial tissue projected forward from the surface of the scar in 4 eyes, and 2 of them also showed radiating retinal striae indicating subretinal fibrosis. In Coats's disease haemorrhages have been noted within the subretinal tissue as well as continuity of retinal vessels with those within the subretinal mass. ${ }^{5}$ In our study none of the eyes showed haemorrhage within the plaque, subretinal haemorrhage, retinal choroidal anasto- 
mosis, pronounced serous retinal detachment, or macular drusen at any stage in a follow-up period of $2 \frac{1}{2}$ to 9 years.

The hard exudate plaques, which measured 0.5 to $1.00 \mathrm{DD}$, showed complete resolution with organisation within 8 to 18 months, which occurred spontaneously in case 3 and as a response to photocoagulation in cases 1,2 , and 4 . A pigment disturbance which may represent pigment epithelial proliferation was observed to develop beneath the resolving plaque in case 1 , and was observed in case 3 at the first examination. In cases 3 and 4 (left eye) resolution and organisation of the foveal plaques preceded the complete absorption of hard exudates elsewhere in the macula. The fluorescein angiography appearance of the scar was consistent with the morphology previously described by us. The early phase of angiography showed a mottled hyperfluorescence from an intact choriocapillaris beneath a normal Bruch's membrane and pigment epithelium showing proliferation and degeneration with loss of pigment content. Discrete residual hyperfluorescence in the late angiograms resembled leakage from the choriocapillaris beneath the pigment epithelium, which was elevated by a serous detachment. Transmitted fluorescence due to depigmentation of pigment epithelium and leakage from diabetic retinal capillary disease within the scar contributed to the late appearance. We could observe no evidence of ingrowing choroidal neovascularisation or serous retinal detachment. ${ }^{3}$

In Dobree's study of the natural history of diabetic maculopathy ${ }^{2}$ there were 14 eyes with plaques 0.5 DD or greater within the macula that were observed over an average interval of $4 \cdot 1$ years. Complete resolution occurred in 7 eyes, leaving a residue of marked pigmentation in 6 eyes and a central fibrous scar in 1 eye. There, was significant visual improvement in 3 eyes, one of which recovered to $6 / 9$ vision, no change in 3 eyes, and a deterioration from $6 / 25$ to $6 / 60$ in the eye showing scar tissue. The natural duration of the plaques was not specifically stated. In 3 of our 5 cases vision remained unchanged (6/60 to counting fingers at 1 metre) when scar tissue developed. Visual prognosis was good when the foveola was unaffected (case 1), and vision recovered from $6 / 120$ to $6 / 15$ in case 2 when the foveola was partly involved.

An understanding of the variable deposition and reabsorption of hard exudate has been derived from observations of circinate deposits which were observed to surround clusters of microaneurysms frequently. ${ }^{7}$ Fluorescein angiography studies ${ }^{8}$ and histochemical studies of hard exudate ${ }^{9} 10$ have provided evidence that most of the deposition results from focal plasma capillary leakage and that the absorption takes place at the periphery where capillaries are less deranged, and by slow removal of the lipid component by phagocytosis. Morphologically in diabetic retinopathy large deposits of hard exudate collect mainly in the outer plexiform layer, surrounded by degenerative neuronal elements, with lipid-laden macrophages within and surrounding the exudate..$^{9-12}$ Degeneration of photoreceptors ${ }^{13}$ and continuity through the external limiting membrane with material beneath the retina have sometimes been observed..$^{14}$ The deep accumulation of hard exudate in juxtaposition to the pigment epithelium may be a circumstance which causes focal alteration, perhaps metaplasia, in the pigment epithelium, which leads to organisation with scar formation. When severe retinal oedema is present, direct apposition of the plaque and the pigment epithelium may be prevented by an extension of the extracellular fluid into the subretinal space, which is difficult to detect biomicroscopically or by fluorescein angiography because of overlying cystoid changes. A reduction in leakage, either spontaneous after progressive capillary obliteration or after photocoagulation, accompanied by absorption of the thin layer of subretinal fluid, would lead to apposition and perhaps initiate the organisation of a deeply situated plaque by the pigment epithelium.

This work was supported in part by National Institute of Health Grant EY 02102-01.

R.S. is in receipt of a University of British Columbia teaching fellowship.

\section{References}

${ }^{1}$ King RC, Dobree, JH, Kok D'A, Foulds WS, Dangerfield WG. Exudative diabetic retinopathy: spontaneous changes and effects of a corn oil diet. Br J Ophthalmol 1963; 47: 666-72.

${ }^{2}$ Dobree JH. Simple diabetic retinopathy. Evolution of the lesions and therapeutic considerations. $\mathrm{Br} J$ Ophthalmol 1970; 54: 1-10.

${ }^{8}$ Begg IS, Rootman J. Clinico-pathological study of an organized plaque in exudative diabetic maculopathy. Can J Ophthalmol 1976; 11: 197-202.

${ }^{4}$ Hogan MJ, Alvarado JA, Weddell JE. Retina. In: Hogan MJ, Alvarado JA, Weddell JE, eds. Histology of the human eye: an atlas and textbook. Philadelphia: Saunders, 1971.

${ }^{5}$ Gass JDM. A fluorescein angiographic study of macular dysfunction secondary to retinal vascular disease $\mathrm{V}$. Retinal telangiectasia. Arch Ophthalmol 1968; 80: 592-605. ${ }^{6}$ Campbell FP. Coats's disease and congenital vascular retinopathy. Trans Am Ophthalmol Soc 1976; 74: 365-421.

'Houston WR, Wise GN. Circinate retinopathy. Arch Ophthalmol 1957; 58: 783-96.

${ }^{8}$ Maumenee AE. Fluorescein angiography in the diagnosis and treatment of lesions of the ocular fundus. Trans Ophthalmol Soc UK 1968; 88: 529-56.

${ }^{9}$ Toussant D, Cogan DG, Kuwabara T. Extravascular 
lesions of diabetic retinopathy. Arch Ophthalmol 1962; 67: 42-7.

${ }^{10}$ Yanko L, Ungar H, Michaelson IC. The exudative lesions in diabetic retinopathy with special regard to the hard exudate. Acta Ophthalmol (Kbh) 1974; 52: 150-60.

${ }^{11}$ Wolter JR, Goldsmith RI, Phillips RL. Histopathology of the star-figure of the macular area in diabetic and angiospastic retinopathy. Arch Ophthalmol 1957; 57: 376-85.
${ }^{12}$ Friedenwald JS. Diabetic retinopathy. Am J Ophthalmol 1970; 33: 1187-99.

${ }^{13}$ Bloodworth JMB. Diabetic retinopathy. Diabetes 1962; 11: 1-22.

${ }^{14}$ Cogan DG. Significance of fundus signs. In: Smith LH, ed. Ophthalmic manifestations of systemic vascular disease: Major problems in internal medicine. Philadelphia: Saunders, 1974: 3. 